\title{
Modeling of Solvolysis Reaction Mechanism for Cation-Olefin Cyclizations
}

\author{
Guillaume Bollot§, Céline C.J. Fouillet, and Jiri Mareda* \\ $\S S C S$ Poster Prize Winner
}

\begin{abstract}
We report on a computational study of cation-olefin cyclization mechanism under solvolytic conditions. The combined explicit-continuum solvent model aimed at providing a complete estimate of media effects is used to investigate the nature, stability, and conformational behavior of cationic species involved in the reaction. The comparison between the explicit-continuum and leaving group models is also provided. Our study of reaction pathways shows that protonated cyclopropane is the key intermediate on the potential energy surface of cationolefin cyclizations.
\end{abstract}

Keywords: Ab initio · Carbocations · Cation-olefin cyclizations · Molecular modeling · Solvent effects

\section{Introduction}

Cation-olefin cyclizations are among the most important and also the most complex carbon-carbon bond forming reactions [1]. A number of enzymes and catalytic antibodies generate the spectacular biosynthetic results often with a very good degree of stereo control via the catalysis of cationic cyclizations. Such catalysis is widespread in the bio-genesis of complex natural products. In the organic synthesis the cationolefin cyclization can be achieved simply by solvolysis of an appropriate substrate. Scheme 1 depicts the results [2] of the cation-olefin cyclization of the substrate 1 which was catalyzed by an antibody as well as subjected to solvolytic conditions. While the products differ for the antibody cata- lyzed (top) and for the solvolysis pathways (bottom), these two reactions are assumed to involve the same key intermediate: the protonated cyclopropane 2 [2][3].

In this work we report on the progress achieved in a computational study of cation-olefin cyclizations under solvolytic conditions. For the potential energy surface investigations it is important a) to model the leaving group by an appropriate functional group and b) to take into account the solvent effects. We describe our approach that takes into account the media effects for solvolysis reactions involving unusual cationic intermediates such as protonated cyclopropanes and hyperconjugatively stabilized ions. We also compare the simple leaving group model with the more elaborate one; the socalled combined explicit-continuum solvent model. The latter is aiming at providing a more complete appreciation of solvent effects upon the carbocation conformations and relative stabilities during the solvolysis reactions.

\section{Solvolysis Models and Computational Methods}

All calculations were carried out using the Gaussian-98 and -03 program suites [4]. The electron correlation was handled with the MP2 perturbation method [5]. The 6-31G* basis set was used in all geometry optimizations. With the exception of the MP4 method, all energies were computed also with the larger and more accurate 6$311+\mathrm{G}^{* *}$ basis set. The nature of all sta-
${ }^{*}$ Correspondence: Dr. J. Mareda University of Geneva Department of Organic Chemistry $\mathrm{CH}-1211$ Geneva 4 Tel.: +41223796099 Fax: +412237932 16

E-Mail: Jiri.Mareda@chiorg.unige.ch

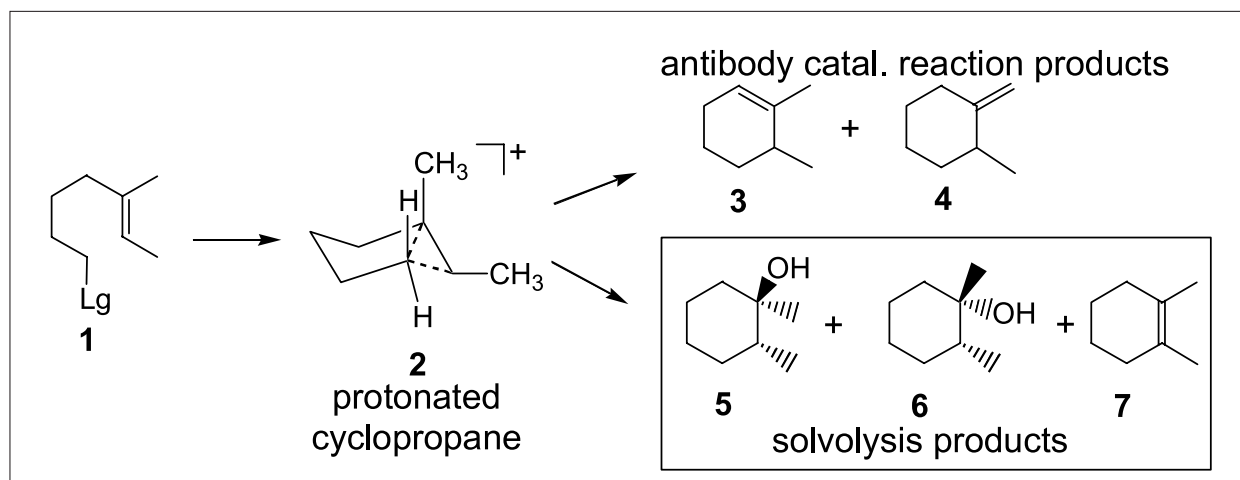

Scheme 1. 
tionary points was examined with frequency calculations, which equally provided zero-point energy (ZPE) corrections [6]. Due to the complexity of the potential energy surfaces associated with this reaction, the majority were also examined by means of intrinsic reaction coordinate [7] which allowed the association of the specific transition state with the corresponding reactant and product. We also tested the ONIOM method for the geometry optimizations of cation-water clusters. This method has been shown to be a powerful tool for the theoretical treatment of larger molecular systems [8]. Within this formalism, the surrounding water molecules were simulated at a lower level of theory, namely, the $3-21 \mathrm{G}$ basis set, while modeling the cationic moiety at a higher level: MP2/6-31G*.

The specific modeling of the solvolysis reaction mechanism was undertaken in two stages. First, the leaving group model was used in which the leaving group was simulated by the protonated alcohol as the reactant of the reaction, becoming a natural water molecule in the cationic product (Scheme 2). Hence, along the reaction coordinate corresponding to the heterolytic cleavage of the carbon oxygen bond, the cation-water complex is formed. The major advantage of this type of simulation is the avoidance of the unrealistic primary carbocation on the reactant side, while simulating the presence of the leaving group along the entire reaction path. This is especially valuable for the investigation of the transition state region.

In the second stage, the solvent effects were taken into account using the combined explicit-continuum solvent model. In this approach, the first solvation shell is simulated with explicit water molecules clustering around the cationic moiety, and their positions as well as their interactions with the substrate are computed at the adequate quantum chemistry level. In addition to the explicit molecules in the immediate vicinity of the reactive intermediates, the second, third and further solvation shells are approximated with the standard DCPM continuum method [9].

\section{Results and Discussion}

The main focus of this contribution being the explicit-continuum solvent model, Fig. 1 shows, mainly for comparison purposes, only several of the salient features of the energy profile for the leaving group modeling. This model has been described in more detail elsewhere [10]. The low activation barrier and the large exothermicity of this reaction are the main characteristics of its energy profile. It also indicates the formation of the two cationic products: the strongly puckered cyclohexyl cation 9

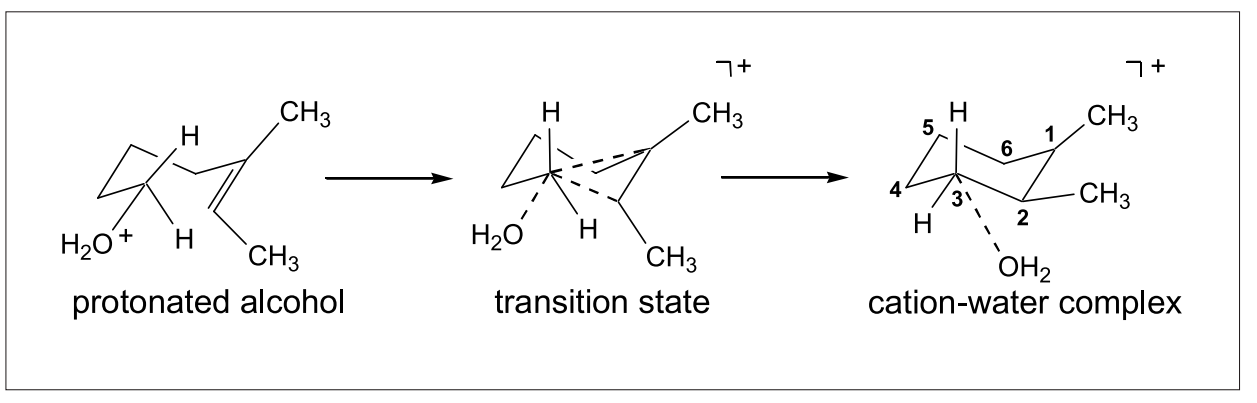

Scheme 2.

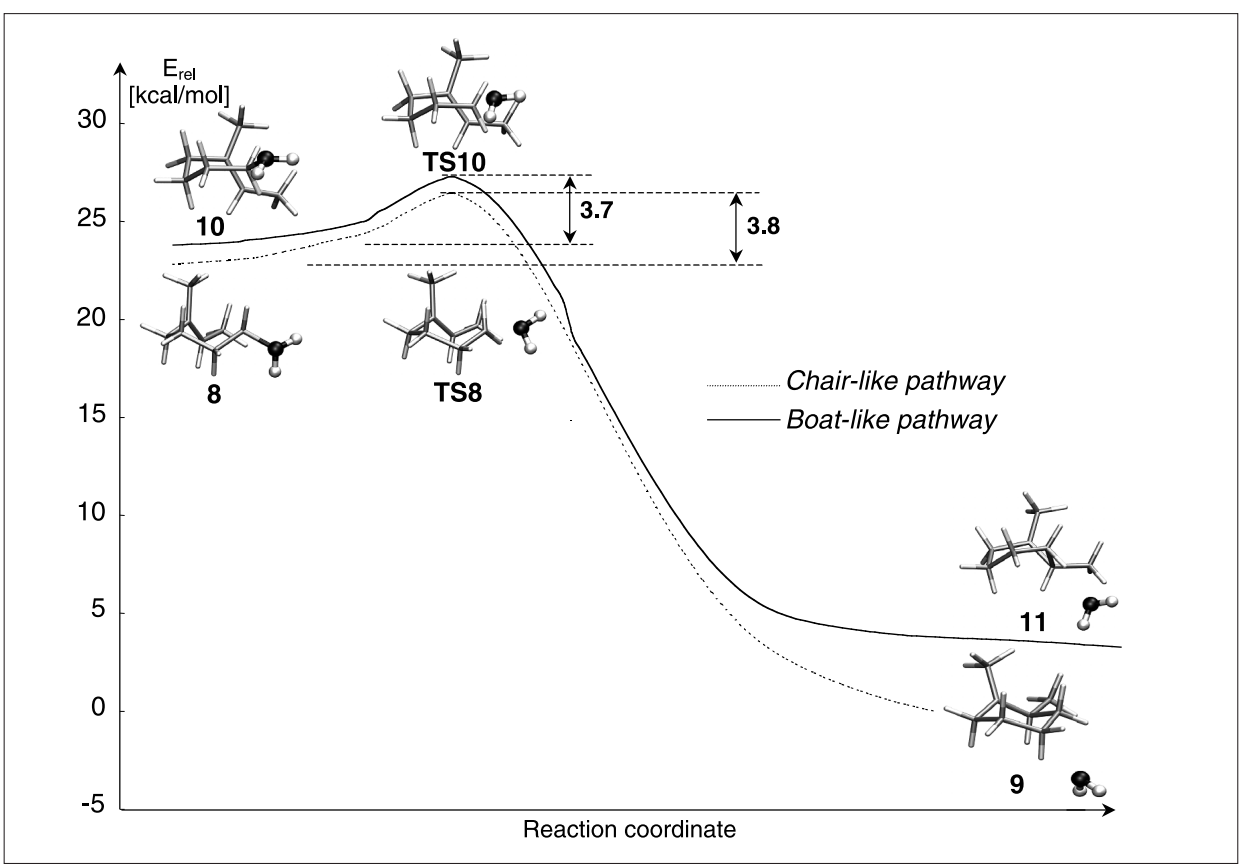

Fig. 1. Leaving group model - the MP2/6-31G* potential energy surface along the intrinsic reaction coordinates of cyclizations for protonated alcohols via the chair- (dotted line) and the boat-like (full line) pathways.

formed via the chair-like pathway, and the protonated cyclopropane $\mathbf{1 1}$ generated via the boat-like mechanism.

One of the main motivations in employing the explicit-continuum solvent model for cation-olefin cyclizations was the need to reexamine the nature of cationic intermediates involved in the solvolysis mechanism and in particular the need to evaluate the stability of protonated cyclopropane 2 . To our satisfaction the cationic products of the cyclization are of the same type as those obtained with the leaving group model. The energy profile of the chair-like pathway (left part of Fig. 2) depicts the formation of the strongly puckered cyclohexyl cation 13. Similar to the formation of ion 9 in the leaving group model, the carbocation $\mathbf{1 3}$ possesses the two strongly elongated carbon-carbon bonds $(\mathrm{C}(2)-\mathrm{C}(3)=1.603 \AA$, $\mathrm{C}(5)-\mathrm{C}(6)=1.580 \AA)[11]$ since they are quasi-aligned with the p-vacant orbital of the cationic center [10][12]. The additional water molecules and the surrounding continuum apparently influenced only marginally the double $\mathrm{C}-\mathrm{C}$ hyperconjugative interactions. The boat-like pathway (Fig. 2) leads to the protonated cyclopropane cation 15 with the two long cyclopropane bonds: $\mathrm{C}(2)-\mathrm{C}(3)=1.788 \AA, \mathrm{C}(1)-\mathrm{C}(3)=1.789 \AA$ [11]. These bonds delimit the symmetrical protonated cyclopropane moiety of ion $\mathbf{1 5}$, which contrast with the pronounced dissymmetry of equivalent bonds in ion $\mathbf{1 1}$ [10]. The structural and electronic characteristics of the transition states differ mainly in one aspect: TS12 and TS14 are late saddle points when compared to TS8 and TS10, which appear early along their respective reaction pathways.

While the comparison of cationic structures shows many similarities in both models, the reaction energy profiles, however, are quite different. For the explicit-continuum model (Fig. 2) the reaction is only weakly exothermic and the activation bar- 


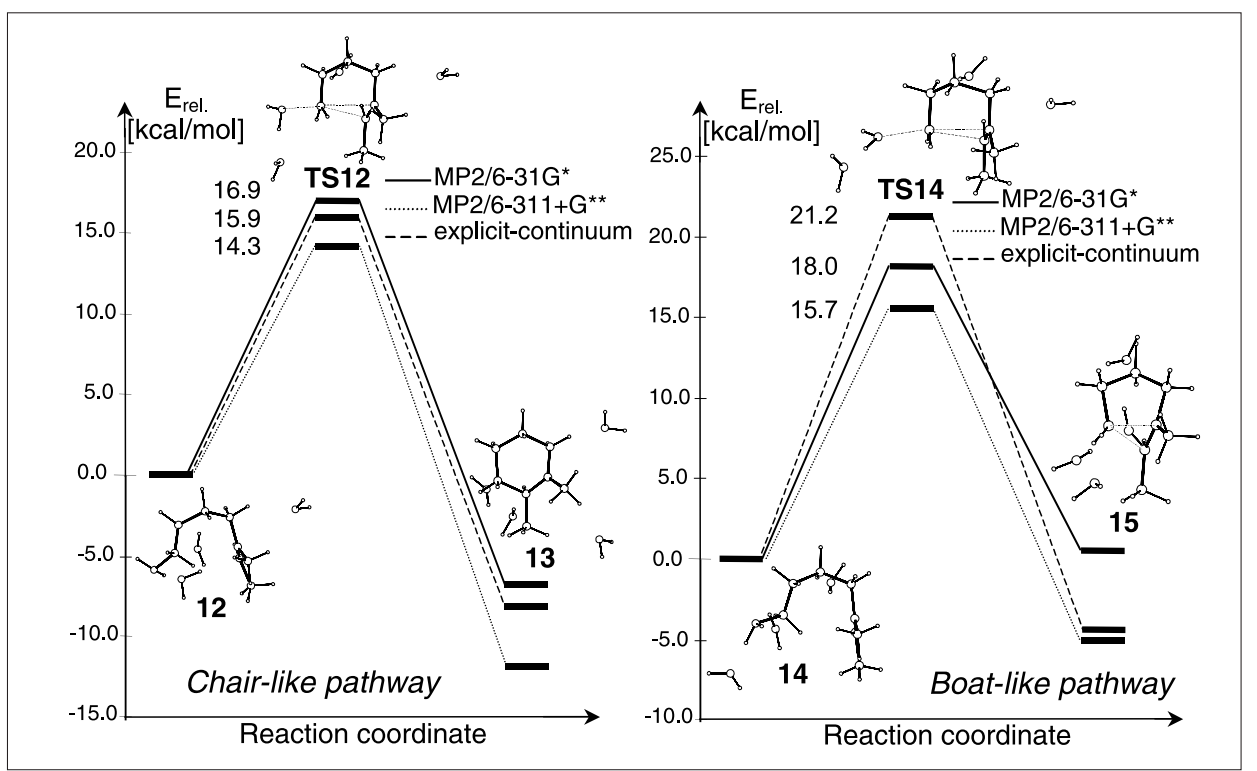

Fig. 2. Explicit-continuum solvent model - schematic energy profile for cation-olefin cyclizations computed at different theory levels. The MP2/6-31G* profile (full line) is based on intrinsic reaction coordinate calculations.

Table 1. Total and relative energies for cation-olefin cyclizations of $\left[\mathrm{C}_{8} \mathrm{H}_{15} \mathrm{OH}_{2}\right]^{+}-3\left(\mathrm{H}_{2} \mathrm{O}\right)$ system at the MP2 level of theory.

\begin{tabular}{|c|c|c|c|c|c|c|}
\hline \multirow[t]{2}{*}{ Species } & \multicolumn{4}{|c|}{ MP2/6-31G*//MP2/6-31G* } & \multicolumn{2}{|c|}{$\mathrm{MP} 2 / 6-311+\mathrm{G}^{\star *} / / \mathrm{MP} 2 / 6-31 \mathrm{G}^{*}$} \\
\hline & $E_{\text {tot }}{ }^{a}$ & $\mathrm{E}_{\mathrm{rel}} \mathrm{b}$ & $Z \mathrm{PE}^{\mathrm{C}}$ & $E_{r e l}(Z P E)^{d}$ & $E_{\text {tot }}^{a}$ & $\mathrm{E}_{\mathrm{rel}}{ }^{\mathrm{e}}$ \\
\hline 12 & -617.29843 & 8.5 & 0.317085 & 9.7 & -617.81377 & 12.6 \\
\hline TS12 & -617.27148 & 25.4 & 0.314002 & 24.6 & -617.79087 & 27.0 \\
\hline 13 & -617.30930 & 1.6 & 0.312554 & 0.0 & -617.83266 & 0.7 \\
\hline 14 & -617.31192 & 0.0 & 0.317538 & 1.5 & -617.82580 & 5.0 \\
\hline TS14 & -617.28331 & 18.0 & 0.315750 & 18.3 & -617.80083 & 20.7 \\
\hline 15 & -617.31120 & 0.5 & 0.314962 & 0.3 & -617.83383 & 0.0 \\
\hline
\end{tabular}

${ }^{a}$ Total electronic energies in Hartree. ${ }^{b}$ Energies relative to 14 in $\mathrm{kcal} / \mathrm{mol} .{ }^{c}$ Energetic correction established at MP2/6-31G* ${ }^{*}$ ZPE scaled by a factor 0.9676 [6]. d Corrected ZPE energies relative to $13 \mathrm{in} \mathrm{kcal} / \mathrm{mol}$. ${ }^{e}$ Energies relative to $15 \mathrm{in} \mathrm{kcal} / \mathrm{mol}$.

Table 2. Total and relative energies for cation-olefin cyclizations of $\left[\mathrm{C}_{8} \mathrm{H}_{15} \mathrm{OH}_{2}\right]^{+}-3\left(\mathrm{H}_{2} \mathrm{O}\right)$ system at the MP4 and CEC (Combined Explicit-Continuum model) MP2 levels of theory.

\begin{tabular}{|c|c|c|c|c|c|}
\hline \multirow[t]{2}{*}{ Species } & \multicolumn{3}{|c|}{ MP4(SDQ)/6-31G*//MP2/6-31G* } & \multicolumn{2}{|c|}{ CEC MP2/6-311+G**//MP2/6-31G* } \\
\hline & $E_{\text {tot }}{ }^{a}$ & $\mathrm{E}_{\mathrm{rel}}^{\mathrm{b}}$ & $\mathrm{E}_{\mathrm{rel}}(\mathrm{ZPE})^{\mathrm{c}}$ & $E_{\text {tot }}{ }^{d}$ & $\mathrm{E}_{\mathrm{rel}} \mathrm{e}^{\mathrm{e}}$ \\
\hline 12 & -617.41293 & 9.5 & 11.9 & -617.89229 & 13.3 \\
\hline TS12 & -617.38702 & 25.8 & 26.2 & -617.86684 & 29.3 \\
\hline 13 & -617.42733 & 0.5 & 0.0 & -617.90464 & 5.6 \\
\hline 14 & -617.42814 & 0.0 & 2.6 & -617.90616 & 4.6 \\
\hline TS14 & -617.39751 & 19.2 & 20.7 & -617.87238 & 25.8 \\
\hline 15 & -617.42273 & 3.4 & 4.4 & -617.91355 & 0.0 \\
\hline
\end{tabular}

a Total electronic energies in Hartree. ${ }^{b}$ Energies relative to 14 in $\mathrm{kcal} / \mathrm{mol} .{ }^{c}$ Energies ZPE correction established at MP2/6-31G ${ }^{*}$ relative to 13 in $\mathrm{kcal} / \mathrm{mol}$. ${ }^{d}$ Total electronic CEC energies in Hartree. ${ }^{e}$ Energies CEC relative to 15 in $\mathrm{kcal} / \mathrm{mol}$. riers for both pathways are much higher than in the leaving group modeling. The chair-like pathway barrier of $15.9 \mathrm{kcal} / \mathrm{mol}$ (dashed line in Fig. 2) is more than $5 \mathrm{kcal} /$ mol lower than that of the boat-like cyclization (barrier of $21.2 \mathrm{kcal} / \mathrm{mol}$ ), indicating clearly the selectivity in favor of the former mechanism. Therefore the formation of $\mathbf{1 3}$ is favored under the kinetic control, while the protonated cyclopropane $\mathbf{1 5}$ is some 6 $\mathrm{kcal} / \mathrm{mol}$ more stable than the chair conformer 13. The predicted selectivity also differs between the two models, since the leaving group modeling suggests no specific selectivity for the cyclization (Fig. 1) and the strongly puckered chair conformer 9 has been predicted to be the more stable carbocation of the two products.

It is interesting to follow the evolution of the relative stabilities of the two cationic cyclization products $\mathbf{1 3}$ and $\mathbf{1 5}$ within the explicit-continuum model, when energies are computed at different levels of theory. At the MP2/6-31G* level, the protonated cyclopropane $\mathbf{1 5}$ is slightly more stable than 13, however, this small difference almost disappears when ZPE correction is applied (Table 1). With the more accurate $6-311+\mathrm{G}^{* *}$ basis set, the ion $\mathbf{1 5}$ remains the more stable product, although only by a weak margin. In the combined explicit-continuum model computed at the MP2/6-311+G** level, the protonated cyclopropane $\mathbf{1 5}$ this time appears clearly much more stabilized than the puckered chair cyclohexyl cation $\mathbf{1 3}$ (compare the right column in Table 2). This shows how important the incorporation of both explicit and continuum solvent effects is for the evaluation of energies for this type of reactions. Surprisingly, the MP4/6-31G* method favors the chair conformer $\mathbf{1 3}$ by some $4.4 \mathrm{kcal} / \mathrm{mol}$, although this result was obtained in the absence of the solvent bulk. Based mainly on the explicit-continuum results listed above, we consider that the protonated cyclopropane cation $\mathbf{1 5}$ is the global minimum on the potential energy surface of products.

We also explored the possibility of reducing the computational cost by using the ONIOM method for geometry optimizations of cation-water clusters. The corresponding energy data listed in Table 3 show a quite satisfactory agreement with results listed in Tables 1 and 2. In the case of the explicit-continuum model the chair conformer $\mathbf{1 3}$ is shown incorrectly as being slightly more stable than ion $\mathbf{1 5}$, showing thereby the limitations of this method for the solvent modeling.

\section{Conclusion}

The method where the solvent effects are taken into account via the combined ex- 
Table 3. Total and relative energies for cation-olefin cyclizations of $\left[\mathrm{C}_{8} \mathrm{H}_{15} \mathrm{OH}_{2}\right]^{+}-3\left(\mathrm{H}_{2} \mathrm{O}\right)$ system at the ONIOM, MP2 and CEC (Combined ExplicitContinuum model) MP2 methods.

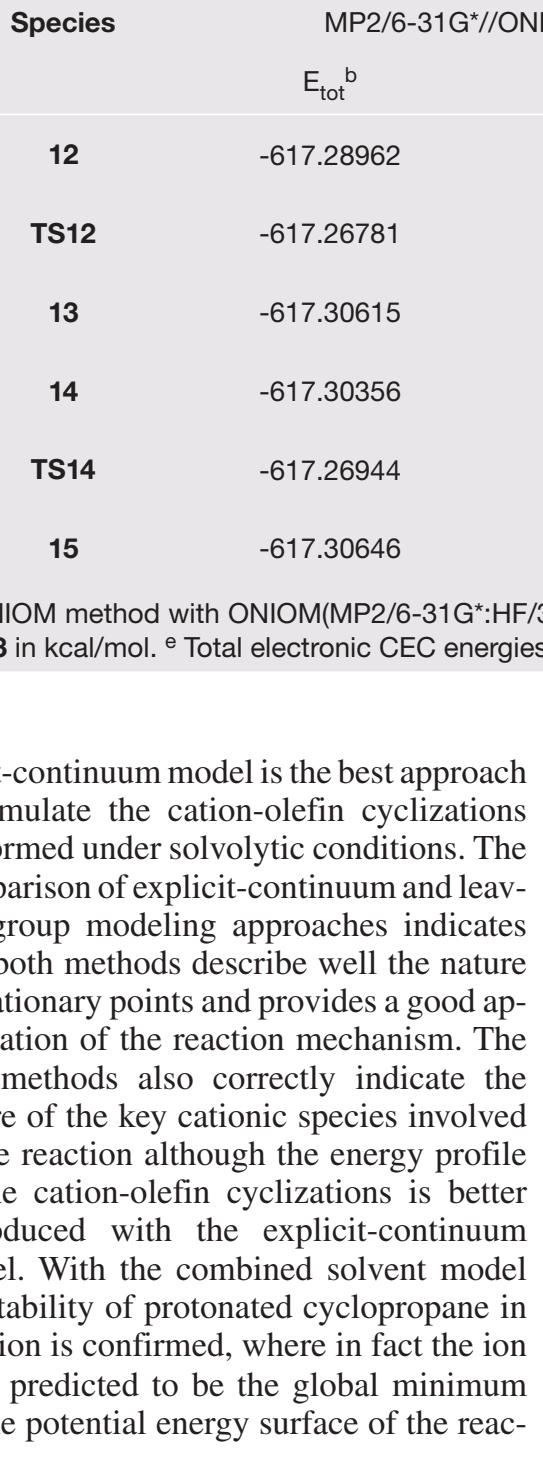

plicit-continuum model is the best approach to simulate the cation-olefin cyclizations performed under solvolytic conditions. The comparison of explicit-continuum and leaving group modeling approaches indicates that both methods describe well the nature of stationary points and provides a good appreciation of the reaction mechanism. The two methods also correctly indicate the nature of the key cationic species involved in the reaction although the energy profile of the cation-olefin cyclizations is better reproduced with the explicit-continuum model. With the combined solvent model the stability of protonated cyclopropane in solution is confirmed, where in fact the ion $\mathbf{1 5}$ is predicted to be the global minimum on the potential energy surface of the reaction.

\section{Acknowledgments}

We are indebted to the Swiss National Foundation (Project No. 21-45790.95) for its financial support. We thank the Swiss Scientific Computing Center for granting us CPU time on their NEC SX-5 computer.

Received: February 4, 2004
[1] I. Abe, M. Rohmer, G.D. Prestwich, Chem Rev. 1993, 93, 2189.

[2] a) T. Li, K.D. Janda, R.A Lerner, Acc. Chem. Res. 1997, 30, 115; b) J. Hasserodt, K.D. Janda, R.A. Lerner, J. Am. Chem. Soc. 2000, 122, 40.

[3] J.K. Lee, K.N. Houk, Angew. Chem., Int. Ed. Engl. 1997, 36, 1003.

[4] M.J. Frisch, G.W. Trucks, H.B. Schlegel, G.E. Scuseria, M.A. Robb, J.R. Cheeseman, V.G. Zakrzewski, J.A. Montgomery, R.E. Stratmann, J.C. Burant, S. Dapprich, J.M. Millam, A.D. Daniels, K.N. Kudin, M.C. Strain, O. Farkas, J. Tomasi, V. Barone, M. Cossi, R. Cammi, B. Mennucci, C. Pomelli, C. Adamo, S. Clifford, J. Ochterski, G.A. Petersson, P.Y. Ayala, Q. Cui, K. Morokuma, D.K. Malick, A.D. Rabuck, K. Raghavachari, J.B. Foresman, J. Cioslowski, J.V. Ortiz, B.B. Stefanov, G. Liu, A. Liashenko, P. Piskorz, I. Komaromi, R. Gomperts, R.L. Martin, D.J. Fox, T. Keith, M.A. Al-Laham, C.Y. Peng, A. Nanayakkara, C. Gonzalez, M. Challacombe, P.M.W. Gill, B.G. Johnson, W. Chen, M.W. Wong, J.L. Andres, M. Head-Gordon, E.S. Replogle, J.A. Pople, Gaussian 98 (Revision A.7), Gaussian, Inc., Pittsburgh PA, 1998.
[5] a) C. Moller, M.S. Plesset, Phys. Rev. 1934, 46, 618; b) R. Krishnan, J.A. Pople, Int. J. Quantum Chem. 1978, 14, 91; c) R. Krishnan, M.J. Frisch, J.A. Pople, J. Chem. Phys. 1980, 72, 4244.

[6] J.B. Foresman, A. Frisch, 'Exploring Chemistry with Electronic Structure Methods', Gaussian Inc. Pittsburgh, 1996.

[7] C. Gonzalez, H.B. Schlegel, J. Phys. Chem. 1990, 94, 5523.

[8] a) S. Dapprich, I. Komáromi, K.S. Byun, K. Morokuma, M.J. Frisch, J. Mol. Struct. Theochem. 1999, 1, 462; b) T. Vreven, K. Morokuma, J. Comp. Chem. 2000, 21, 1419.

[9] a) V. Barone, M. Cossi, J. Tomasi, J. Comp. Chem. 1998, 19, 404; b) V. Barone, M. Cossi, J. Phys. Chem. A 1998, 102, 1995.

[10] C.C.J. Fouillet, J. Mareda, J. Mol. Struct. Theochem. 2002, 589, 7.

[11] For the atom numbering see Scheme 2.

[12] A. Rauk, T.S. Sorensen, C. Maerker, J.W.M. Carneiro, S. Sieber, P.v.R. Schleyer, J. Am. Chem. Soc. 1996, 118, 3761. 